\title{
ATLAS Detector Data Processing on the Grid
}

\author{
Alexei A. Klimentov, Pavel L. Nevski, Jonas Strandberg, Junji Tojo, Alexandre V. Vaniachine, and \\ Rodney Walker on behalf of the ATLAS Collaboration
}

\begin{abstract}
The ATLAS detector is in the second year of continuous LHC running. A starting point for ATLAS physics analysis is data reconstruction. Following the prompt reconstruction, the ATLAS data are reprocessed, which allows reconstruction of the data with updated software and calibrations providing coherence and improving the quality of the reconstructed data for physics analysis.

The large-scale data reprocessing campaigns are conducted on the Grid. Computing centers around the world participate in reprocessing providing tens of thousands of $\mathrm{CPU}$-cores for a faster throughput. Reprocessing relies upon underlying ATLAS technologies providing reproducibility of results, scalable database access, orchestrated workflow and performance monitoring, dynamic workload sharing, and petascale data integrity control. These technologies are also empowering ATLAS physics and subsystem groups in further data processing steps on the Grid.

We present the experience of large-scale data reprocessing campaigns and group data processing on the Grid.
\end{abstract}

Index Terms-Data processing, distributed computing, grid computing, petascale computing.

\section{INTRODUCTION}

$T_{p}^{n}$ he "raw" data from the ATLAS detector (Fig. 1) are processed to produce the reconstructed data for physics analysis. The distributed multi-tier data processing architecture handles petascale data flow. Fig. 2 shows data processing flow of raw event and conditions/calibrations data used in reconstruction operations.

The "first-pass" processing of the raw event data at the ATLAS Tier-0 site provides promptly the data for quality assessment and analysis. Later, the quality of the reconstructed data is improved by optimizing further software algorithms

Summary of the paper received May 10, 2011. This work was supported in part by the U.S. Department of Energy, Division of High Energy Physics, under Contract DE-AC02-06CH11357.

A. A. Klimentov is with the Department of Physics, Brookhaven National Laboratory, Upton, NY 11973 USA (e-mail: klimentov@bnl.gov).

P. L. Nevski is with the Department of Physics, Brookhaven National Laboratory, Upton, NY 11973 USA (e-mail: nevski@bnl.gov).

J. Strandberg is with the Division of Particle and Astroparticle Physics, KTH Royal Institute of Technology, SE-100 44 Stockholm, Sweden (e-mail: jostran@kth.se).

J. Tojo is with the High Energy Accelerator Research Organization (KEK), Tsukuba, Japan (e-mail: Junji.Tojo@cern.ch).

A. V. Vaniachine is with the Division of High Energy Physics, Argonne National Laboratory, Argonne, IL 60439 USA (phone: 630-252-1878; fax: 630-252-6169; e-mail: vanachine@anl.gov).

R. Walker is with the Physics Department, LMU Munich University, Munich, Germany (e-mail: Rodney.Walker@physik.uni-muenchen.de). and conditions/calibrations data. For data processing with improved software and conditions/calibrations (reprocessing) we use distributed computing resources.

ATLAS distributed computing resources are an order of magnitude larger than the resources at the Tier-0 alone. ATLAS uses three Grids (each with a different interface) split in ten "clouds" organized as large computing centres with tape data storage (Tier-1 sites) each associated with 5-6 other computing centres (Tier-2 sites). Plus more than a hundred of ATLAS Tier-3 sites used for the physics analysis. All these computing resources are used in further data processing steps following the ATLAS detector data reconstruction.

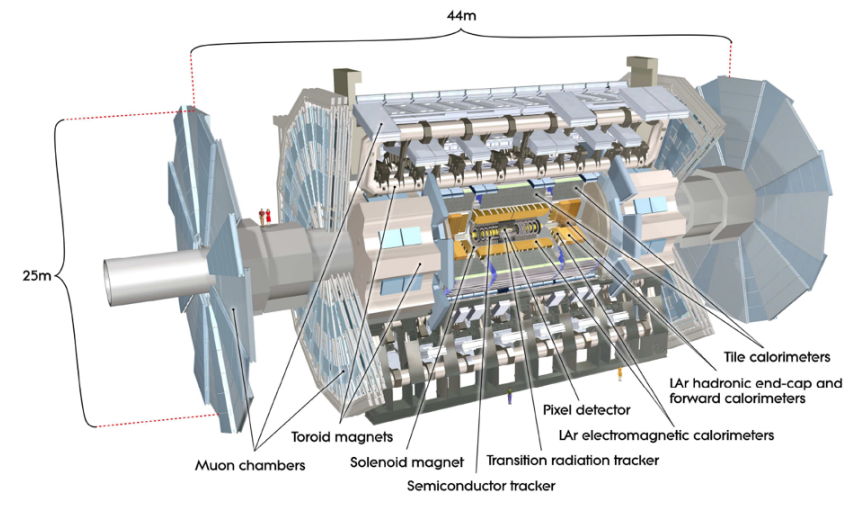

Fig. 1. The data for reconstruction come from the ATLAS detector [1].

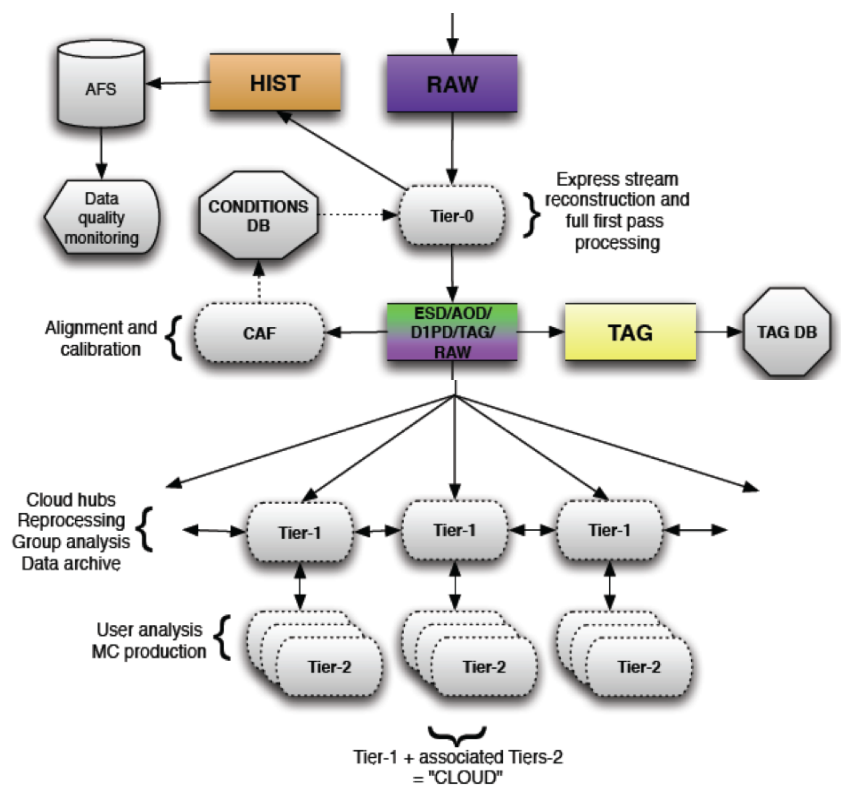

Fig. 2. Simplified flow of raw event and conditions/calibrations data used in reconstruction at the Tier- 0 site at CERN (top) and on the Grid at the Tier-1 sites (bottom). 


\section{REQUIREMENTS AND TECHNOLOGIES}

\section{A. Reproducibility of Results}

We developed Sites Validation tools that ensure that all Grid sites produce numerically identical outputs for the same raw data inputs. Sites Validation ensures that the same ATLAS software and conditions/calibrations versions are used worldwide and excludes discrepancies due to heterogeneity of the Grid such as site-specific CPU (AMD/Intel), system libraries, batch systems, etc. Sites Validation also enables software release validation with a large-scale data sample revealing rare software bugs.

\section{B. Scalable Database Access}

To prevent scalability problems in database access on the Grid we developed a technology for access to the conditions/calibrations data, which is similar to the raw event data and software release distribution on the Grid. The Database Release technology integrates in a single dataset all conditions/calibrations data required for reconstruction [2].

\section{Workflow Orchestration}

Leveraging the underlying technology [3], our stable but flexible production system ensures efficient usage of computing resources for complex data processing on tens of thousands of CPU-cores. During reprocessing the system monitors site performance and supports dynamic workload sharing minimizing the reprocessing duration.

\section{Data Integrity}

To facilitate physics discoveries, the reprocessing must minimize event losses. This is assured by automated resubmission of the failed data processing jobs, which excludes transient failures. The events that cannot be reconstructed during the reprocessing campaign are recovered promptly in a dedicated post-processing step using an updated software release and/or conditions/calibrations.

\section{Petascale Data Processing Experience}

Thanks to the technologies described in the previous section, no events were lost during the main ATLAS reprocessing campaign of the 2010 data that reconstructed on the Grid more than 1 PB of data with $0.9 \cdot 10^{9}$ events.

Later silent data corruption was detected in six events, which corresponds to event losses at the $10^{-8}$ level. During the "first-pass" processing the event losses have been at the $10^{-4}$ level, which was tolerated to deliver the reconstructed data promptly [4]. In reprocessing, a reduction in the event losses by four orders of magnitude was achieved at the expense of the CPU losses - transient failures recovery increased the average number of attempts for every job to 1.2. Following the S-Curve dependence (Fig. 3), most of the CPU losses were caused by two (known) bugs that in a small subset of tasks resulted in endless loops at the end of data reconstruction jobs.

Our experience shows that correcting silent data corruption in a distributed petascale event store is prohibitively costly. To assure scalability, the data corruption must be detected at the spot. In a petascale event store, every layer of services should not assume that the underlying layer never provide corrupted

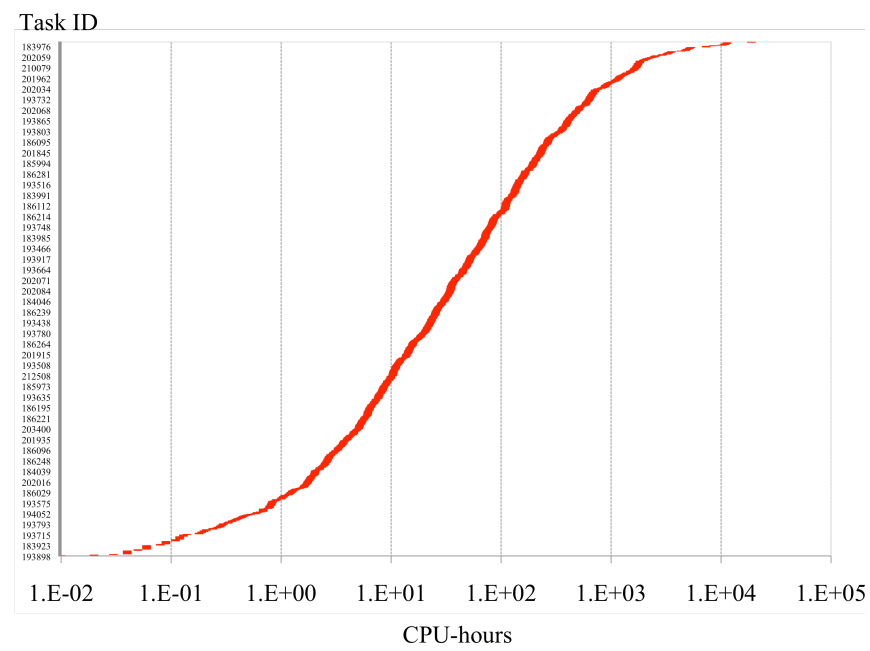

Fig. 3. Data processing tasks ordered by CPU losses show that top 21 tasks lost more than $1 \%$ of total losses each.

or inconsistent data. We must have redundancy in order to detect and recover from data corruption errors.

\section{GRoup DATA PRocessing}

The reprocessing technologies empowered further data processing steps on the Grid performed by dozens of ATLAS physics groups with coordinated access to computing resources worldwide. In 2010, Group Production activities consumed most of the ATLAS Grid computing resources.

These technologies were also adopted for the trigger reprocessing, which is performed to validate new trigger menus or other trigger changes critical during 2011 LHC operations.

\section{CONCLUSION}

ATLAS technologies for Grid data processing coped well with the first petabytes of LHC data. Since the start of LHC data taking ATLAS successfully completed eight reprocessing campaigns on the Grid. The average number of attempts required to eliminate transient failures on the Grid provides a benchmark for further performance improvements. Initial operational experience demonstrates that the petascale event store architecture requires end-to-end data integrity strategy.

\section{ACKNOWLEDGMENT}

We wish to thank all our colleagues who contributed to ATLAS reprocessing activities.

\section{REFERENCES}

[1] The ATLAS Collaboration, G Aad et al., (2008, August). "The ATLAS experiment at the CERN Large Hadron Collider," J. Inst., [Online]. Vol. 3, S08003, Available: http://iopscience.iop.org/1748-0221/3/08/S08003

[2] M. Borodin, P. Nevski, A. Vaniachine, "Scaling up ATLAS Database Release technology for the LHC long run," J. Phys.: Conf. Ser., to be published. Available: http://cdsweb.cern.ch/record/1322655

[3] T. Maeno. (2008). "PanDA: distributed production and distributed analysis system for ATLAS" J. Phys.: Conf. Ser., [Online]. Vol. 119, 062036, Available: http://iopscience.iop.org/1742-6596/119/6/062036

[4] S. Oda. (2011, Apr 19). "Prompt calibration, assessment of data quality and data processing." Poster ATL-PHYS-SLIDE-2011-163. [Online]. Available: $\underline{\text { http://cdsweb.cern.ch/record/1345770 }}$ 